\title{
Nomogram for Prediction of the International Study Group of Liver Surgery (ISGLS) Grade B/C Posthepatectomy Liver Failure in HBV-related Hepatocellular carcinoma Patients: an external validation and prospective application study
}

Jiazhou Ye ( $\nabla$ yejiazhou2019@163.com )

Guangxi Medical University

\section{Rong-yun Mai}

Guangxi Cancer Hospital and Guangxi Medical University Affiliated Cancer Hospital

Wei-xing Guo

Shanghai East Hospital

\section{Yan-yan Wang}

Beijing Cancer Hospital

\section{Liang Ma}

Guangxi Cancer Hospital and Guangxi Medical University Affiliated Cancer Hospital

\section{Bang-de Xiang}

Guangxi Cancer Hospital and Guangxi Medical University Affiliated Cancer Hospital

\section{Wan-Yee Lau}

Prince of Wales Hospital and Community Health Services

\section{Shu-qun Cheng}

Shanghai East Hospital

\section{Lequn Li}

Guangxi Cancer Hospital and Guangxi Medical University Affiliated Cancer Hospital

\section{Research}

Keywords: Hepatocellular carcinoma, Hepatitis B virals, Posthepatectomy liver failure, Nomogram

Posted Date: April 1st, 2020

DOI: https://doi.org/10.21203/rs.3.rs-20106/v1

License: @ (i) This work is licensed under a Creative Commons Attribution 4.0 International License. Read Full License 
Version of Record: A version of this preprint was published at BMC Cancer on October 28th, 2020. See the published version at https://doi.org/10.1186/s12885-020-07480-2. 


\section{Abstract}

Background \& Aims: To develop a nomogram for predicting the International Study Group of Liver Surgery (ISGLS) grade B/C posthepatectomy liver failure (PHLF) in hepatitis B virus (HBV)-related hepatocellular carcinoma (HCC) patients.

Methods: Patients initially treated with hepatectomy were included. Univariate regression analysis and stochastic forest algorithm were applied to extract the core indicators and reduce redundancy bias. The nomogram was then constructed by using multivariate logistic regression, and validated in internal and external cohorts, and a prospective clinical application.

Results: There were 900, 300 and 387 participants in training, internal and external validation cohorts, with the morbidity of grade B/C PHLF were $13.5 \%, 11.6 \%$ and $20.2 \%$, respectively. The nomogram was generated by integrating preoperative total bilirubin, platelet count, prealbumin, aspartate aminotransferase, prothrombin time and standard future liver remnant volume, then achieved good prediction performance in training (AUC $=0.868$, $95 \% \mathrm{Cl}=0.808-0.880)$, internal validation ( $\mathrm{AUC}=0.868,95 \% \mathrm{Cl}=0.794-0.916)$ and external validation cohorts (AUC $=0.820,95 \% \mathrm{Cl}=0.756-0.861$ ), with well-fitted calibration curves. Negative predictive values were significantly higher than positive predictive values in training cohort $(97.6 \%$ vs. $33.0 \%)$, internal validation cohort $(97.4 \%$ vs. $25.9 \%)$ and external validation cohort (94.3\% vs. $41.1 \%)$, respectively. Patients who had a nomogram score $<169$ or $\geqq 169$ were considered to have low or high risk of grade B/C PHLF. Prospective application of the nomogram accurately predicted grade B/C PHLF in clinical practise.

Conclusions: The nomogram has a good performance in predicting ISGLS grade B/C PHLF in HBV-related HCC patients and determining appropriate candidates for hepatectomy.

\section{Background:}

Hepatocellular carcinoma (HCC) is the sixth most common malignancy and the fourth leading cause of cancerrelated death worldwide.[1] Hepatectomy is the most effective treatment for early-stage HCC patients,[2] and selective intermediate-stage and advanced-stage HCC patients with resectable tumors and moderate liver function.[3] Advances surgical techniques and management have greatly improved the safety and postoperative outcomes over the past few decades;[4] however, the International Study Group of Liver Surgery (ISGLS) grade $\mathrm{B} / \mathrm{C}$ posthepatectomy liver failure (PHLF) remains a serious complication, which is a predominant cause of postoperative mortality.[5, 6]

Incidence of PHLF as reported in literature widely ranges from $1.2 \%-32 \%$ attributing to diverse etiological and pathogenic liver characteristics and surgical procedures. [6, 7] Independent risk factors of PHLF can be grouped into three categories[5, 8]: 1) Patient-related factors including age, sex, comorbidities such as malnutrition, diabetes mellitus, cardiopulmonary, renal or cerebral dysfunction; 2) liver disease-related factors including hepatitis B/C, steatosis, cholangitis, alcoholic liver disease and cirrhosis; 3 ) surgery-related factors including future liver remnant volume (FLRV), excessive intraoperative blood loss, prolonged operation time, and ischemiareperfusion injury resulting from Pringle's manoeuver manipulation. In particular, as a major cause to promote decompensate liver cirrhosis and dysfunction, chronic hepatitis B is highly prevalent and associated with $70 \%-90 \%$ of HCC cases in the Asia-Pacific region.[9] 
Accurate prediction of PHLF is of primary concern for determining the feasibility of hepatectomy for HCC.[5, 8] Child-Pugh grade,[10] model for end-stage liver disease (MELD),[11] albumin-bilirubin (ALBI),[12, 13] plateletalbumin-bilirubin (PALBI) and aspartate aminotransferase to platelet ratio index (APRI)[14] are commonly conventional scores used for evaluating PHLF, nevertheless their predictive performance remains controversial due to inherent limitations. Child-pugh grade is the most widely used for evaluating compensate liver function and has been incorporated into surgical treatment algorithms.[11] However, subjective and unquantifiable variables usually complicate Child-pugh grade: serum bilirubin level of $55 \mu \mathrm{mol} / \mathrm{L}$ has the same influence on Child-pugh grade as $550 \mu \mathrm{mol} / \mathrm{L}$ due to arbitrary thresholds for continuous variables; there is no clear guideline for distinguishing mild or moderate ascites, and the influence of diuretic therapy on grading ascites remains unclear; sedatives therapy frequently mislead encephalopathy.[15] MELD was developed to evaluate acute liver failure mortality risk and rank candidates for transplantation,[16, 17] which was also good at determining increased PHLF morbidity and mortality risk when a MELD score > 8 on postoperative day 5.[5] However, MELD has a poor performance at preoperative predicting PHLF.[5, 6, 11] ALBI statistical eliminates subjective observation and assesses liver function and overall survival compared favorably with Child-pugh grade in four geographical and etiological HCC patient groups.[18] A preoperative ALBI score predicting PHLF was more accurately than Child-pugh grade, MELD and indocyanine green retention at 15 minutes (ICG-15).[12, 13] However, when patients with hyperbilirubinemia were divide into the ALBI grade 3, patients with obstructive jaundice may have better liver function and prognosis than patients with jaundice caused by decompensate liver dysfunction, which significantly misleading the grading of ALBI.[19, 20] Blood platelet (PLT) counts as a surrogate marker of portal hypertension was added to ALBI to develop the PALBI, which predicting survival in HCC patients across treatment modalities including hepatectomy was better than ALBI and MELD. Nonetheless, further research is necessary as few studies have been done evaluating use of PALBI for predicting PHLF. APRI is noninvasive and reliable for evaluating liver fibrosis and cirrhosis,[21, 22] meanwhile a preoperative APRI score $\geq$ 10 have a high risk of PHLF in HCC patients.[16] However, APRI only includes two quantitative variables and has no ceiling effect. In general, these conventional scores were primarily designed for assessing liver function or other purposes rather than predicting PHLF. Moreover, when they were used for predicting PHLF none of these scores comprehensively considers patient-related, liver-related, and surgical-related risk factors.

As an evidence-based model, nomogram has been proposed as an alternative tool for therapy risk individualized estimation in clinical application. $[23,24]$ This study aimed to establish a nomogram to predict grade B/C PHLF risk for HBV-HCC patients.

\section{Methods}

\section{Patient population}

This study was conducted retrospectively in HBV-related HCC patients who were initially treated with hepatectomy. The training and internal cohorts consisted of patients treated at the Guangxi Medical University Cancer Hospital (GXMUCH) between October 11th, 2013 and December 21st, 2017. The external cohort consisted of patients treated at the Eastern Hepatobiliary Surgery Hospital (EHBH) between September 14th, 2009 and January 22th, 2018. In addition, patients would receive hepatectomy as the initial treatment, were prospectively recruited from GXMUCH between December 22th, 2017 and June 21st, 2018 for evaluation of the nomogram in clinical application. This study was approved by the Institutional Ethics Committees of the two hospitals. 
The inclusion criteria were as follows: (1) aged 18-75 years, (2) positive for hepatitis B surface antigen, (3) preoperative Eastern Cooperative Oncology Group (ECOG) performance score 0-2, (4) preoperative Child-pugh A/B liver function, and (5) histological diagnosis of HCC. Patients who had other simultaneous malignancies, hepatitis $\mathrm{C}$, tumor rapture, extrahepatic metastasis, cardiopulmonary, renal or cerebral dysfunction, and underwent preoperative anticancer treatments were excluded.

\section{Definitions}

ISGLS PHLF was diagnosed with an increased serum international normalized ratio (INR) and concomitant hyperbilirubinaemia after postoperative day 5.[5] The severity of PHLF was graded as: grade A PHLF required no specific treatment; grade B PHLF required essential non-invasive treatment, such as fresh frozen plasma, administration of albumin and daily diuretics; grade C PHLF required invasive procedures including mechanical ventilation, hemodialysis or extracorporeal liver support. The standardized future liver remnant (sFLR) = FLR/estimated total liver volume (eTLV) was used to represent the percentage of postoperative residual liver. The equation calculating $\operatorname{eTLV}\left(\mathrm{cm}^{3}\right)=706.2 \times$ body surface area $(\mathrm{BSA})\left(\mathrm{m}^{2}\right)+2.4 ; \mathrm{BSA}\left(\mathrm{m}^{2}\right)=0.00607 \times$ height $(\mathrm{cm})+$ $0.0127 \times$ weight $(\mathrm{kg})-0.0698$ for men and $\mathrm{BSA}\left(\mathrm{m}^{2}\right)=0.00586 \times$ height $(\mathrm{cm})+0.0126 \times$ weight $(\mathrm{kg})-0.0461$ for women.[25]

\section{Preoperative examination and surgical procedure}

Preoperative general characteristics, laboratory biochemistry data (including liver and renal function tests, hepatitis immunology and serum a-fetoprotein level), radiological data (including abdominal contrast-enhanced CT or MRI scan, and chest radiograph), surgical data (including operation time, intraoperative blood loss, intraoperative transfusion, sFLR) were routinely collected. sFLR was determined by three-dimensional technology using software DEMedical (version3.1, Shenzhen, China). Surgical procedures have been described in a previous report.[3] Pringle's manoeuver was applied to occlude the liver blood. Electrosurgical instruments or clampcrushing method was performed to carry out liver parenchymal transection. Histopathological examination was routinely conducted by three pathologists on all surgical specimens. The main outcome observed was the PHLF morbidity and mortality.

\section{Study design and statistical analyses}

Flow chart of the study design is shown in Fig. 1. A stratified random grouping method was performed to randomly assign and divide patients into training cohort and internal validation cohort at a ratio of 3:1. In training cohort, logistic univariate analysis was used to identify independent risk indicators of grade B/C PHLF and correlation analysis was performed to eliminate data redundancy and excessive false positives. When correlation analysis indicated non-independence from univariate analysis, indicators were classified according to clinical significance in seven groups with different meanings (liver synthesis ability, metabolism ability, HBV activity status, liver inflammation, compensate cirrhotic liver function, coagulation function, and surgery-related factors). Stochastic According to the forest algorithm, indexes with the highest weight (at least $>20$ ) in each category were extracted and incorporated into the subsequent logistic multivariate regression model. A nomogram was formulated using the RMS package in $\mathrm{R}$ version 3.3.2. The predictive performance of the nomogram was measured using receiver operating characteristic (ROC) curve and compared with conventional scores. Calibration plots methods evaluated the goodness of fit for the nomogram. Yoden index of the ROC curve from training cohort was calculated to set the diagnostic threshold. The diagnostic errors were displayed by correcting the curve. Area under the curve (AUC) represents the misdiagnosis threshold. Correspondingly, the 
confidence interval of diagnosis is expressed in abscissa of area beyond the $95 \%$ misdiagnosis threshold. For efficacy evaluation of the nomogram in prospective clinical application, total points of predictions was calculated for each patient, meanwhile statistical indicators including precision, recall, accuracy and F1 balance were calculated to evaluate the diagnostic ability.

Data analysis was performed using SPSS (Version 23.0, IBM, New York, USA) and R software (Version 3.2.2, Institute for Statistics and Mathematics, Vienna, VIC, Austria). Normally distributed continuous data are expressed as mean (s.d.) and compared using an unpaired. Two-tailed t-test Values with a non-normal distribution are expressed as median (IQR 25-75) and were compared using Mann-Whitney U test. Categorical data are shown as frequency and proportion and were compared using the $\chi^{2}$ test.

\section{Results}

\section{Clinicopathologic characteristics}

During the study period, $1200 \mathrm{HBV}$-related HCC patients from GXMUCH met the inclusion criteria were included and randomly assigned to a training cohort $(n=900)$ and an internal validation cohort $(n=300)$ at a ratio of 3:1. Besides, $387 \mathrm{HBV}$-related HCC patients from EHBH met the inclusion criteria were included in an external validation cohort (Fig. 1). Baseline clinicopathologic characteristics are listed in Supplemental Table 1.

The incidences of grade B/C PHLF were $13.5 \%, 11.6 \%$ and $20.2 \%$ in the training cohort, internal cohort and external cohort, respectively (Supplemental Table 1). The postoperative mortality rate of entire participants at GXMUCH was $1.75 \%$ (21 patients): 13 patients died as a result of grade C PHLF following multiple organ failure, 8 patients died due to sepsis or severe pneumonia. The postoperative mortality rate of participants from EHBH was $0 \%$. In participants for prospective clinical application of the nomogram, grade B PHLF occurred in 14 patients $(11.7 \%)$ and grade C PHLF occurred in 4 patients (3.3\%).

\section{Independent risk indicator of ISGLS grade B/C PHLF}

In training cohort, independent risk indicators of grade B/C PHLF was identified by logistic univariate analysis (Fig. 2A) and data redundancy and excessive false positives were eliminated by correlation analysis (Fig. 2B). According to the forest algorithm, serum HBV-DNA loads with weights of 2.19 were excluded (Fig. 2C). Multivariate analysis for a stepwise removal of variables was then done, and the results reported as odds ratios with $95 \% \mathrm{Cl}$, total bilirubin (T-Bil), platelet (PLT) count, prealbumin (PA), aspartate aminotransferase (AST), prothrombin time (PT), and sFLR were identified independently influenced on grade B/C PHLF (Table 1). Then, the second correlation test revealed that not significant independence among these six independent indicators, which are able to be incorporated into the nomogram (Fig. 2D). 
Table 1

Multivariable logistic regression analyses of grade B/C PHLF in the training cohort

\begin{tabular}{|llll|}
\hline Variables & $\boldsymbol{\beta}$ & Odds ratio & P value \\
\hline T-Bil $(\mu \mathrm{mol} / \mathrm{L})$ & 0.040 & $1.041(1.015,1.068)$ & 0.002 \\
\hline $\mathrm{PLT}\left(\times 10^{9} / \mathrm{L}\right)$ & -0.010 & $0.990(0.987,0.993)$ & $<0.001$ \\
\hline $\mathrm{PA}(\mathrm{mg} / \mathrm{L})$ & -0.005 & $0.995(0.992,0.999)$ & 0.019 \\
\hline AST $(\mathrm{U} / \mathrm{L})$ & 0.004 & $1.004(1.000,1.008)$ & 0.035 \\
\hline $\mathrm{PT}(\mathrm{s})$ & 0.318 & $1.375(1.144,1.652)$ & $<0.001$ \\
\hline sFLR $(\%)$ & -0.067 & $0.936(0.926,0.949)$ & $<0.001$ \\
\hline $\begin{array}{l}\text { Abbreviations: TBIL, total bilirubin; PLT, platelet; PA, prealbumin; AST, aspartate aminotransferase; PT, } \\
\text { prothrombin time; SFLR: standard future liver remnant. }\end{array}$ & \\
\hline
\end{tabular}

\section{Development and Validation of a grade B/C PHLF-Predicting nomogram}

Based on the multivariate logistic analysis (Table 1), a nomogram integrating T-Bil, PLT, PA, AST, PT and sFLR was developed (Fig. 3A). ROC curve was applied to evaluate accuracy of the grade B/C PHLF prediction. The area under the ROC curve (AUC) of the nomogram was 0.868 (95\%Cl: $0.808-0.880)$ in training cohort, 0.868 (95\%Cl: $0.794-0.916)$ in internal validation cohort, and 0.820 (95\% Cl: $0.756-0.861)$ in external validation cohort (Fig. 3B). Differences among the three cohorts were not statistically significant. In training cohort, the optimal cutoff value of total points to predict grade B/C PHLF was determined to be 169 , with a sensitivity, specificity, positive predictive value, and negative predictive value of $88.4 \%, 72.1 \%, 33.0 \%$, and $97.6 \%$, respectively. Bootstrap validation results showed good performance, with a sensitivity, specificity, positive predictive value, and negative predictive value of $84.8 \%, 70.0 \%, 25.9 \%$, and $97.4 \%$ in internal validation; and $83.3 \%, 69.9 \%, 41.1 \%$, and $94.3 \%$ in external validation cohort, respectively (Table 2). Calibration curves assessing risk and analyzing consistency of results showed good agreement for probability of grade B/C PHLF between the actual observation and prediction in training cohort $\left(R^{2}=0.992\right)$, internal validation $\left(R^{2}=0.977\right)$ and external validation cohorts $\left(R^{2}=0.946\right)$ (Fig. 3C-E). 
Table 2

Accuracy of the prediction score of the Nomogram for estimating the risk of grade B/C PHLF incidence

\begin{tabular}{|c|c|c|c|}
\hline \multirow[t]{2}{*}{ Variable } & \multicolumn{3}{|l|}{ Value $(95 \% \mathrm{Cl})$} \\
\hline & Training cohort & $\begin{array}{l}\text { Internal validation } \\
\text { cohort }\end{array}$ & $\begin{array}{l}\text { External validation } \\
\text { cohort }\end{array}$ \\
\hline Area under ROC curve & $\begin{array}{l}0.868(0.808 \text { to } \\
0.880)\end{array}$ & 0.868 (0.794 to 0.916$)$ & $0.820(0.756$ to 0.861$)$ \\
\hline Cutoff score & 169 & 169 & 169 \\
\hline Sensitivity, \% & 88.4 (81.0 to 93.3$)$ & 84.8 (67.3 to 94.3$)$ & 83.3 (72.8 to 90.5$)$ \\
\hline Specificity, \% & 72.1 (68.8 to 75.2 ) & 70.0 (64.1 to 75.4$)$ & $69.9(64.4$ to 74.9$)$ \\
\hline Positive predictive value, $\%$ & 33.0 (28.0 to 38.5$)$ & 25.9 (18.2 to 35.4$)$ & 41.1 (33.5 to 49.3$)$ \\
\hline $\begin{array}{l}\text { Negative predictive value, } \\
\%\end{array}$ & 97.6 (95.9 to 98.6$)$ & 97.4 (93.7 to 99.0$)$ & 94.3 (90.3 to 96.8$)$ \\
\hline Positive likelihood ratio & 3.17 (2.79 to 3.62$)$ & 2.83 (2.24 to 3.58$)$ & 2.77 (2.27 to 3.37$)$ \\
\hline Negative likelihood ratio & $0.16(0.10$ to 0.26$)$ & $0.22(0.10$ to 0.49$)$ & $0.24(0.14$ to 0.39$)$ \\
\hline
\end{tabular}

Further, calculations of objectivity evaluation of diagnostic confidence interval revealed that total points of diagnostic errors with 95\% Cl were concentrated in 175 (ranged 158-220) in training cohort; concentrated in 170 (ranged 155-210) in internal validation cohort and concentrated in 176 (ranged 144-240) in external validation cohort (Fig. 4A), respectively. The sizes of confidence intervals among the three cohorts were very similar and the positions of the concentrated total points were close to the best cutoff value of 169. Confidence interval is considered to be prediction of low risk of grade B/C PHLF when total points fall below this range, while to be prediction of high risk of grade B/C PHLF when total points beyond this range. However, when total points fall within this range, the prediction results should be carefully considered.

\section{Comparison of predictive accuracy for grade B/C PHLF among the nomogram and conventional scores}

When compared with conventional scores, the nomogram had greater discriminatory performance for predicting grade B/C PHLF than Child-pugh grade, MELD, ALBI, PALBI and APRI in training cohort, internal validation cohort and external validation cohort (Fig. 4B, Table 3), which was not significantly influenced by inherent heterogeneity in different cohorts. 
Table 3

Discriminatory performance of conventional scores and the nomogram for predicting grade B/C PHLF

\begin{tabular}{|c|c|c|c|c|c|c|c|c|c|}
\hline & \multicolumn{3}{|c|}{ Training Cohort } & \multicolumn{3}{|c|}{ Internal validation Cohort } & \multicolumn{3}{|c|}{ External validation Cohort } \\
\hline & AUC & $95 \% \mathrm{Cl}$ & $\begin{array}{l}\mathrm{P} \\
\text { value }\end{array}$ & AUC & $95 \% \mathrm{Cl}$ & $\begin{array}{l}P \\
\text { value }\end{array}$ & AUC & $95 \% \mathrm{Cl}$ & $\begin{array}{l}P \\
\text { value }\end{array}$ \\
\hline Child-Pugh & 0.616 & $\begin{array}{l}0.558- \\
0.674\end{array}$ & $<0.001$ & 0.608 & $\begin{array}{l}0.502- \\
0.715\end{array}$ & 0.043 & 0.541 & $\begin{array}{l}0.467- \\
0.615\end{array}$ & 0.267 \\
\hline MELD & 0.647 & $\begin{array}{l}0.597- \\
0.697\end{array}$ & $<0.001$ & 0.627 & $\begin{array}{l}0.523- \\
0.731\end{array}$ & 0.017 & 0.601 & $\begin{array}{l}0.535- \\
0.667\end{array}$ & 0.006 \\
\hline ALBI & 0.689 & $\begin{array}{l}0.635- \\
0.743\end{array}$ & $<0.001$ & 0.667 & $\begin{array}{l}0.574- \\
0.760\end{array}$ & 0.002 & 0.620 & $\begin{array}{l}0.551- \\
0.689\end{array}$ & 0.001 \\
\hline PALBI & 0.634 & $\begin{array}{l}0.580- \\
0.687\end{array}$ & $<0.001$ & 0.779 & $\begin{array}{l}0.711- \\
0.847\end{array}$ & $<0.001$ & 0.668 & $\begin{array}{l}0.603- \\
0.732\end{array}$ & $<0.001$ \\
\hline APRI & 0.741 & $\begin{array}{l}0.692- \\
0.789\end{array}$ & $<0.001$ & 0.734 & $\begin{array}{l}0.640- \\
0.827\end{array}$ & $<0.001$ & 0.626 & $\begin{array}{l}0.560- \\
0.692\end{array}$ & 0.001 \\
\hline Nomogram & 0.868 & $\begin{array}{l}0.808- \\
0.880\end{array}$ & $<0.001$ & 0.868 & $\begin{array}{l}0.794- \\
0.916\end{array}$ & $<0.001$ & 0.820 & $\begin{array}{l}0.756- \\
0.861\end{array}$ & $<0.001$ \\
\hline
\end{tabular}

\section{Prospective clinical application of the nomogram to predict grade B/C PHLF}

In order to further evaluate the ability of predicting grade B/C PHLF in clinical application, the nomogram was applied to predict whether grade B/C PHLF occurred in 120 individual HBV-related HCC patients who would receive hepatectomy in $\mathrm{GXCH}$. As a result, we accurately predicted that 85 patients would not have grade $\mathrm{B} / \mathrm{C}$ PHLF, while 16 patients would; the remaining 19 cases were misjudged with total points were within 165-197 (Supplemental Table 2). All miscalculations have been re-evaluated and incorrect predicted total points (ranged 165-197) are fully contained within 158-220. The results of empirical evaluation of confidence interval help to improve the practicability of the nomogram and support the scientific of the study design. In addition, the predictive performance of the nomogram for judging non-occurrence of grade B/C PHLF is good in clinical practice, with a precision of 0.977 , a recall of 0.833 , an accuracy of 0.947 , and a F1balanced Score of 0.899 .

\section{Discussion}

In this study, after eliminating data redundancy and excessive false positives (Fig. 2), a nomogram was developed by integrating five essential preoperative serum laboratory biochemistries (T-Bil, PLT, PA, AST, PT) and sFLR from different categories with clinical significance comprehensively indicating compensate liver function and the percentage of postoperative remain liver (Table 1). Then a graphical and easy-to-use tool was applied for individualized predicting grade B/C PHLF in HBV-HCC patients (Fig. 3A). This nomogram displayed a good accuracy of prediction for grade B/C PHLF (Fig. 3B) and good agreement between probability and actual observation in training cohort, internal validation cohort and external validation cohort (Fig. 3C-E). Besides, this nomogram had greater predicative performance than conventional scores (Fig. 4A, Table 3). Further, calculations 
of predictive value $(95 \% \mathrm{Cls})$ of the nomogram revealed that negative predictive values were significantly higher than positive predictive values in training cohort ( $97.6 \%$ vs. $33.0 \%)$, internal validation cohort $(97.4 \%$ vs. $25.9 \%)$ and external validation cohort ( $94.3 \%$ vs. $41.1 \%$ ) (Table 3 ), respectively, potentially demonstrated that negative predictive values could be precise for predicting grade B/C PHLF. Considering the PHLF can indeed be misdiagnosed, objective evaluation of the diagnostic ability of the nomogram and identification of confidence intervals were conducted. Results from feedback error diagnosis distribution curves revealed that actual distribution of total points for error prediction with 95\% Cl was concentrated in 175 (158-220), 170 (155-210) and 176 (144-240) in training cohort, internal validation and external validation cohorts, respectively, and were very close to the best cutoff value of 169 (Fig. 4A). Such that doctors have more flexibility when using the diagnostic results. Confidence interval is considered to be prediction of low risk of grade B/C PHLF when total points fall below this range and high risk of grade B/C PHLF when total points beyond this range. While total points fall within this range, the prediction results should be carefully considered and further confirmed through other evaluations such as ICG-15 retention or computer residual liver imaging volumetry. In this study, clinical use of the nomogram was evaluated in $120 \mathrm{HBV}$-related HCC patients who will receive hepatectomy. We found that preoperative application of this nomogram had good predictive performance for acutely judging grade B/C PHLF did not occured in 85 patients; and occurred in 16 patients; misjudged in 19 cases with total points were fall within 165-197, which were all fall within the $95 \% \mathrm{Cl}$ of diagnostic errors between 158-220 (Supplemental Table 2). The scoring range of misjudged cases was consistent with the objective evaluation, which supports the scientific nature of this study and guides further flexible application of this nomogram. In addition, this nomogram had a good predictive performance for judging non-occurrence of grade B/C PHLF in clinical practice, which was consistent with negative predictive values analysis.

A growing body of researches confirmed that major hepatectomy and insufficient sFLR was associated with high risk of PHLF. Our result is consistent with previous findings revealed that sFLR in this nomogram is of great importance for predicting grade B/C PHLF and adopted SFLR as an important predictive indicator of grade B/C PHLF in our model. Recently, two radiomics-based nomograms based on portal-phase computed tomography or ultrasound were established to predict PHLF,[26,27] however, they didn't considered the influence of sFLR on PHLF and integrated this indicator. Moreover, considering mild-moderate liver dysfunction after liver surgery is very common, these nomograms remains to be further validated for predicting ISGLS grade B/C PHLF, particular in HBV-related HCC patients.

This study remains some limitations. First, all of the study participants were associated with HBV, while in most western countries and Japan, the majority of HCC cases are related to alcoholic liver disease or HCV. Therefore, further validation is required from other etiological populations. Second, the reliability of the nomogram remains to be further confirmed by conducting prospective and multicenter validation studies with expanding study participants. Moreover, advanced imaging scans and ICG-15 retention to estimate PHLF might be taken into further consideration to improve the diagnostic value.

\section{Conclusions}

By comprehensively integrating five essential preoperative serum laboratory biochemistries (T-Bil, PLT, PA, AST, PT) and sFLR with different clinical significance comprehensively indicating the compensate liver function and the percentage of remain liver after hepatectomy, a novel nomogram was generated for individualized predicting ISGLS grade B/C PHLF in HBV-HCC patients. The results of internal and external validations demonstrated that 
this nomogram had good predicative performance. Prospective clinical application of this nomogram proposed an accurate judgment of non-occurrence of ISGLS grade B/C PHLF. It potentially provides an alternative tool for determining HBV-HCC patients with low risk of ISGLS grade B/C PHLF are appropriate candidates for hepatectomy.

\section{Abbreviations}

HCC, Hepatocellular carcinoma; ISGLS, International Study Group of Liver Surgery; PHLF, Post-hepatectomy liver failure; sFLR, standard future liver remnant; MELD, Model for end-stage liver disease; ALBI, Albumin-bilirubin; PALBI, Platelet-albumin-bilirubin; APRI, Aspartate aminotransferase to platelet ratio index; ECOG, Eastern Cooperative Oncology Group

\section{Declarations}

\section{Ethics approval and consent to participate}

This study was approved and supervised by the Clinical Research Ethics Committee of the Affiliated Tumor Hospital of Guangxi Medical University.

\section{Consent for publication}

It is applicable for publication.

\section{Availability of data and materials}

All data are available within the article and supplementary files, or available from the authors upon request.

\section{Competing interests}

The authors declare that they have no competing interests.

\section{Funding}

This work was supported by the grants from Youth Project of Natural Science Foundation of China (No:81803007); Youth Project of Natural Science Foundation of Guangxi (No:2018GXNSFBA281030); the Key Project of Natural Science Foundation of China (No:81730097); grants from the Science Fund for Creative Research Groups (No: 81521091); the Chang Jiang Scholars Programme (2013) of China Ministry of Education; the National Key Basic Research Programme "973 project" (No:2015CB554000); the Shanghai Municipal Health Bureau (No: SHDC12015106); and the Shanghai Health and Family Planning commission smart medical special project (No:2018ZHYL0212); branch project of the Major National Science and Technology projects (No:2012X10002010001009); branch project of the National high technology research and development programme "863 project" (No:2015AA020103)

\section{Authors' contributions}

J.Y. and R.M. contributed equally to this work; J.Y., R.M., W.G. and Y.W. designed the research study; L.M., B.X., W.L. and S.C. contributed new reagents and analytic tools; J.Y., R.M. and L.L. analyzed the data; and J.Y. and 
R.M. wrote the manuscript. All authors have read and approve the final manuscript.

Acknowledgments:

We sincerely appreciated Professor. Shuqun Cheng and Weixing Guo provided study patients for the external validation.

\section{References}

1. Uttley L, Indave BI, Hyde C, White V, Lokuhetty D, Cree I: Invited commentary - WHO classification of tumors: How should tumours be classified? Expert consensus, systematic reviews or both. Int J Cancer 2020 .

2. Villanueva A: Hepatocellular Carcinoma. N Engl J Med 2019, 380:1450-1462.

3. Jian-hong Z, Yang K, Wen-feng G, et al.: Hepatic resection associated with good survival for selected patients with intermediate and advanced-stage hepatocellular carcinoma. Ann Surg 2014, 260.

4. Emmanuel M, Martin H, Michael S, et al.: Guidelines for Perioperative Care for Liver Surgery: Enhanced Recovery After Surgery (ERAS) Society Recommendations. World J Surg 2016, 40.

5. Schreckenbach T, Liese J, Bechstein WO, Moench C: Posthepatectomy liver failure. Dig Surg 2012, 29:79-85.

6. Rahbari Nuh N, James GO, Robert P, et al.: Post-hepatectomy haemorrhage: a definition and grading by the International Study Group of Liver Surgery (ISGLS). HPB (Oxford) 2011, 13.

7. Catherine P, Sylvie J, Didier D, et al.: Prospective validation of the "fifty-fifty" criteria as an early and accurate predictor of death after liver resection in intensive care unit patients. Ann Surg 2009, 249.

8. van den Broek Maartje A J, Olde Damink Steven W M, Dejong Cornelis H C, et al.: Liver failure after partial hepatic resection: definition, pathophysiology, risk factors and treatment. Liver Int 2008, 28.

9. Man-Fung Y, Jin-Lin H, Anuchit C: Hepatocellular carcinoma in the Asia pacific region. J Gastroenterol Hepatol 2009, 24.

10. Jordi B, Morris S: Management of hepatocellular carcinoma: an update. Hepatology (Baltimore, Md.) 2011, 53.

11. Alessandro C, Giorgio E, Matteo C, et al.: Recovery from liver failure after hepatectomy for hepatocellular carcinoma in cirrhosis: meaning of the model for end-stage liver disease. J Am Coll Surg 2006, 203.

12. Y-Y W, J-H Z, Z-Y S, et al.: Albumin-bilirubin versus Child-Pugh score as a predictor of outcome after liver resection for hepatocellular carcinoma. Br J Surg 2016, 103.

13. Heng Z, Xin Y, Qing-Long L, Qing-Xiang Z, Li X, Yu W: A Comparative Study of Albumin-Bilirubin Score with Child-Pugh Score, Model for End-Stage Liver Disease Score and Indocyanine Green R15 in Predicting Posthepatectomy Liver Failure for Hepatocellular Carcinoma Patients. Digestive diseases (Basel, Switzerland) 2018, 36.

14. Tsuyoshi I, Takahiro U, Shigekazu T, et al.: A simple, noninvasively determined index predicting hepatic failure following liver resection for hepatocellular carcinoma. J Hepatobiliary Pancreat Surg 2009, 16.

15. Li X, Zhen-Wei P, Min-Shan C, et al.: Prognostic nomogram for patients with unresectable hepatocellular carcinoma after transcatheter arterial chemoembolization. J Hepatol 2015, 63.

16. Ganne-Carrié N, Layese R, Bourcier V, et al.: Nomogram for individualized prediction of hepatocellular carcinoma occurrence in hepatitis C virus cirrhosis (ANRS C012 CirVir). Hepatology 2016, 64. 
17. François D, Dominique V: Assessment of the prognosis of cirrhosis: Child-Pugh versus MELD. J Hepatol 2005, 42 Suppl.

18. Russell W, Erick E, Richard F, et al.: Model for end-stage liver disease (MELD) and allocation of donor livers. Gastroenterology 2003, 124.

19. Teh-la H, Han-Chieh L, Cheng-Yuan H, et al.: The model for end-stage liver disease based cancer staging systems are better prognostic models for hepatocellular carcinoma: a prospective sequential survey. Am J Gastroentero/2007, 102.

20. Johnson Philip J, Sarah B, Chiaki K, et al.: Assessment of liver function in patients with hepatocellular carcinoma: a new evidence-based approach-the ALBI grade. J Clin Onco/ 2015, 33.

21. Lau W, Leung K, Leung T W, et al.: A logical approach to hepatocellular carcinoma presenting with jaundice. Ann Surg 1997, 225.

22. Po-Hong $L$, Chia-Yang $H$, Cheng-Yuan $H$, et al.: ALBI and PALBI grade predict survival for HCC across treatment modalities and BCLC stages in the MELD Era. J Gastroenterol Hepato/ 2017, 32.

23. Chun-Tao W, Greenson Joel K, Fontana Robert J, et al.: A simple noninvasive index can predict both significant fibrosis and cirrhosis in patients with chronic hepatitis C. Hepatology (Baltimore, Md.) $2003,38$.

24. Castéra L, Vergniol J, Foucher J, et al.: Prospective comparison of transient elastography, Fibrotest, APRI, and liver biopsy for the assessment of fibrosis in chronic hepatitis C. Gastroenterology 2005, 128.

25. Cieslak Kasia P, Runge Jurgen H, Michal H, Jaap S, Bennink Roelof J, van Gulik Thomas M: New perspectives in the assessment of future remnant liver. Dig Surg 2014, 31.

26. Cai W, He B, Hu M, et al.: A radiomics-based nomogram for the preoperative prediction of posthepatectomy liver failure in patients with hepatocellular carcinoma. Surg Onco/2019, 28.

27. Hu H, Han H, Han XK, Wang WP, Ding H: Nomogram for individualised prediction of liver failure risk after hepatectomy in patients with resectable hepatocellular carcinoma: the evidence from ultrasound data. Hao Hu;Hong Han;Xi Kun Han;Wen Ping Wang;Hong Ding 2018, 28.

\section{Figures}




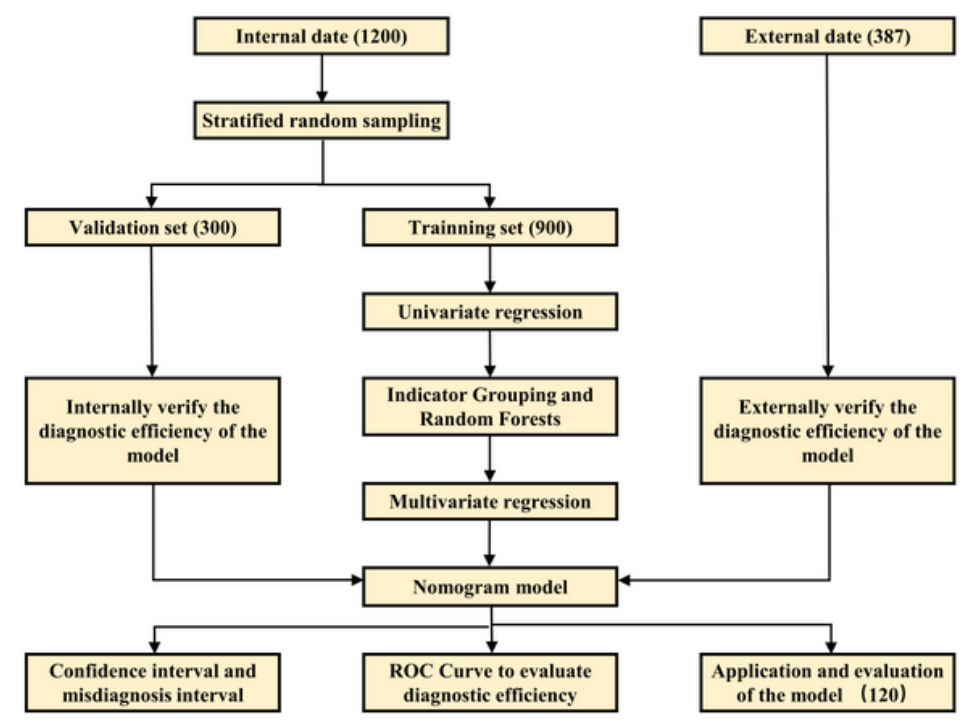

Figure 1

Flow chart of the study design 
A

Variable

$\mathrm{HBV}-\mathrm{DNA},=2000 / 2000(\mathrm{IU} / \mathrm{ml}$

PIT ( $\times 109 /$ )

TBL ( $(\mu \mathrm{mol} / \mathrm{L})$

PA (mgL)

ALB (gL)

ALT (UL)

AST (U/L)

PT (s)

CSPH, Yes / NO

Ascites, Yes / NO

Tumor size (cm)

Tumor number, Multiple/ Single

Portal invasion or Etrahlepatic spread, Yes / No

BCLC stage, $0 / \mathrm{A} / \mathrm{B} / \mathrm{C}$

Operation tinc (min)

Blood loss, $=400 /<400$ (mL)

Blood transfusion, Yes $/ \mathrm{No}$

Cirrhosis, Yes / N

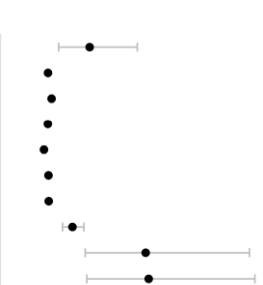

$\mathbf{B}$
0.625
-0.006
0.06
-0.06
-0.05
0.004
0.00
0.4
1.11
1.134
0.08
0.83
0.45
0.32
0.00
0.917
1.054
0.04
0.685
B

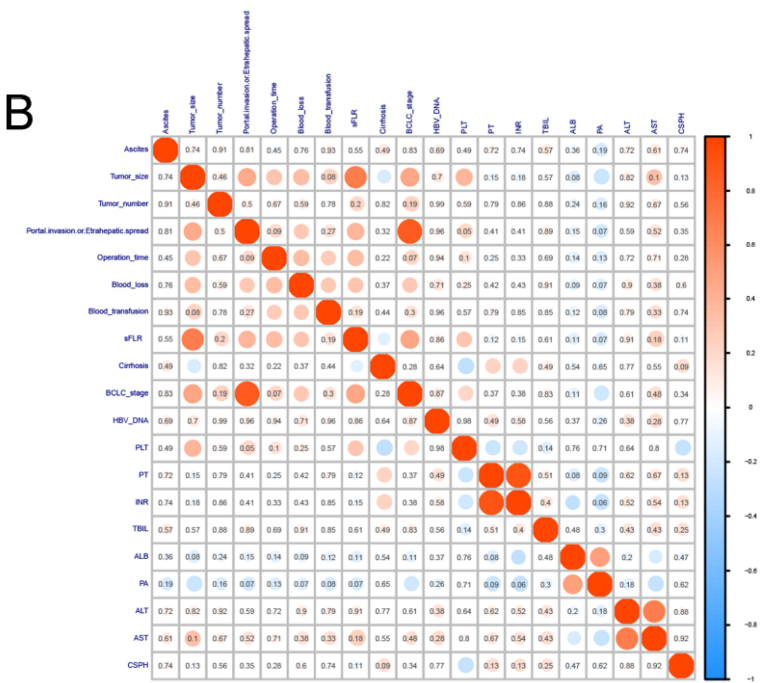

D

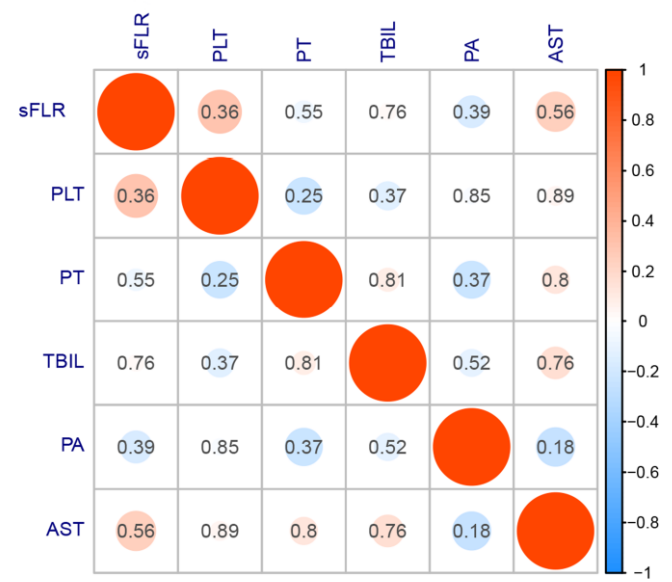

Figure 2

Univariable logistic regression analyses to identify predictors of Grade B/C PHLF in patients with HBV-related $\mathrm{HCC}$ in the training cohort. Forest maps show the risk ratios of indicators. 2B: Correlation analysis among indicators significantly related with grade B/C PHLF by logistic univariate analysis. Colors from red to blue indicate a correlation from positive to negative. The values represent the significant $P$ values of the correlations, indicating the parts of correlations are significant. 2C: The importance of the Stochastic Forest algorithm based on grouping indexes. Logistic univariate significant indicators were divided into seven groups according to clinical significance and a random forest model was constructed for each group of indicators to predict grade B/C PHLF risk. The bars represent the importance of each indicator; the red bars represent the most important indicators of each group. 2D: There is no correlation among the indicators after redundancy removal by grouping stochastic forest algorithm. Colors from red to blue indicate a correlation from positive to negative. The values inside the circle represent the significant $P$ values of the correlations, indicating the correlations among all indicators are not significant. 
$A_{\text {Points }}$

PLT count $(\times 10 \%)$

T-Bil ( $\mu \mathrm{mol} / \mathrm{L})$

$\mathrm{PA}(\mathrm{mg} / \mathrm{L})$

PT (s)

AST (U/L)

$\operatorname{sFLR}(\%)$

Total Points

Risk of Grade B/C PHLF

C

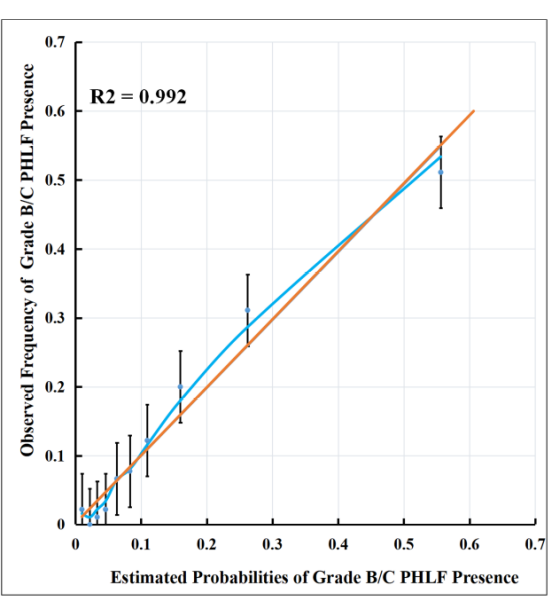

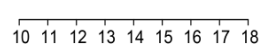

$0 \quad 100200300 \quad 400$

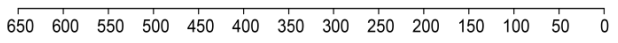

$$
\begin{array}{lllllllll}
\hline 0 & 20 & 40 & 60 & 80 & 100 & 120 & 140 & 160
\end{array}
$$

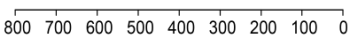

$100 \quad$\begin{tabular}{lllllllllllllll}
\hline & 9 & 1 & 1 & 1 & 1 & 1 & 1 & 1 & 1 & 1 & 1 & 1 & 1 & 1
\end{tabular}

\begin{tabular}{lllllllllllllll}
\hline 0 & 20 & 40 & 60 & 80 & 100 & 120 & 140 & 160 & 180 & 200 & 220 & 240 & 260 & 280
\end{tabular}

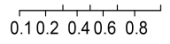

$\mathrm{D}$

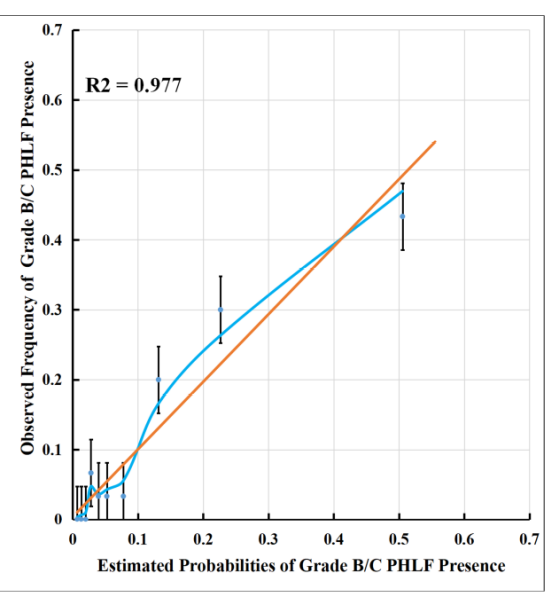

B

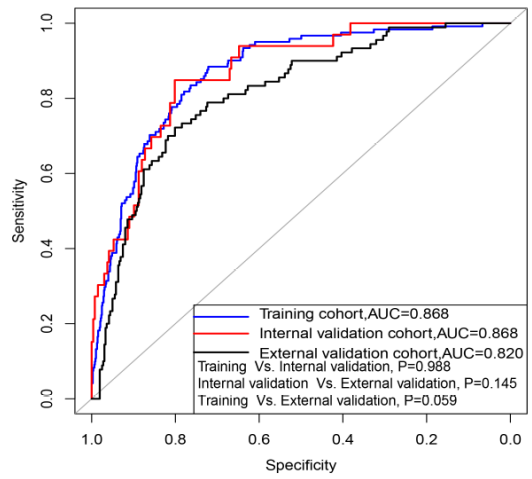

$E$

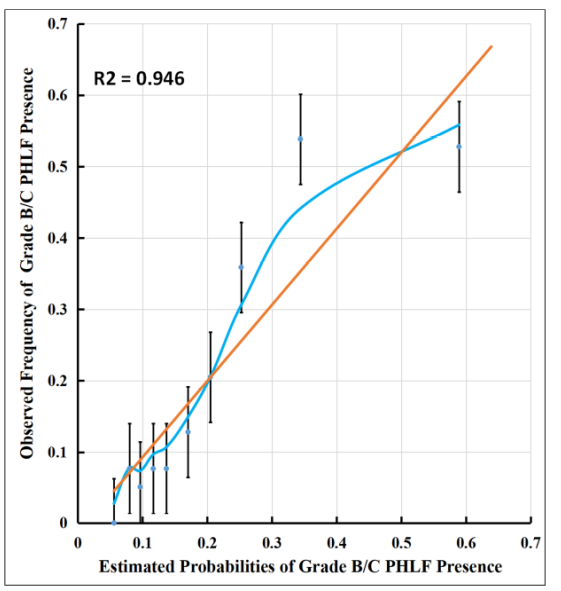

\section{Figure 3}

A. Nomogram for predicting grade B/C PHLF in HBV-related HCC patients. To use the nomogram, find the position of each variable on the corresponding axis, draw a line to the points axis for the number of points, add the points from all of the variables, and draw a line from the total points axis to determine the grade B/C PHLF probabilities at the lower line of the nomogram. B: Receiver operating characteristic (ROC) curves for the nomogram in predicting grade B/C PHLF. Calibration plots show the relationship between the predicted probabilities based on the nomogram and actual values $\mathbb{C}$. training cohort, $D$. internal validation cohort, $E$. external validation cohort. Nomogram-predicted probability of grade B/C PHLF is plotted on the $x$-axis, and the actual probability is plotted on the $y$-axis. 
A

a

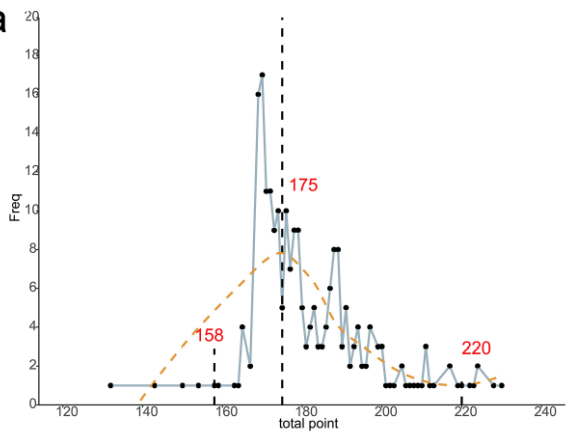

B

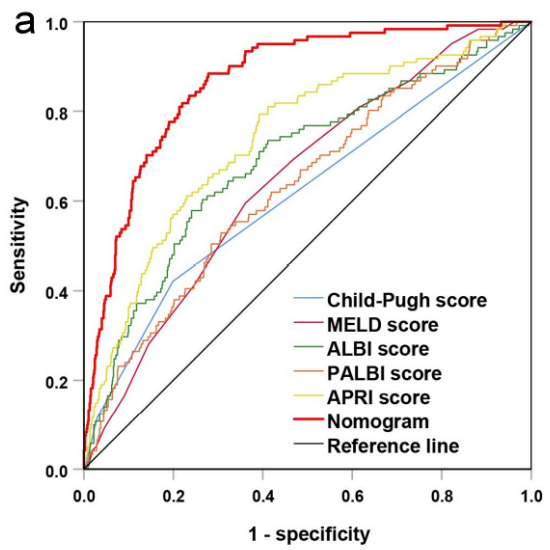

b
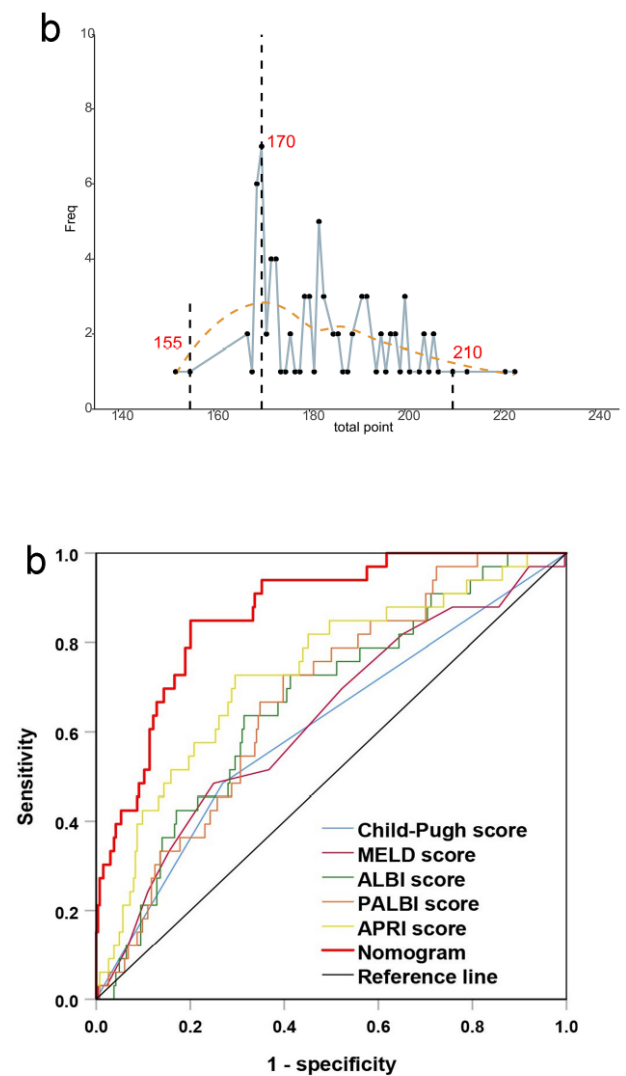

C

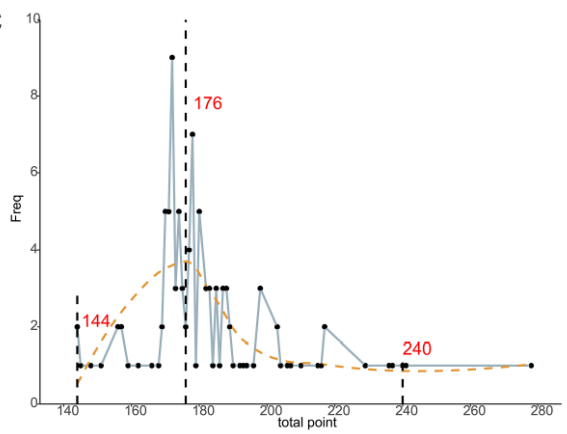

C

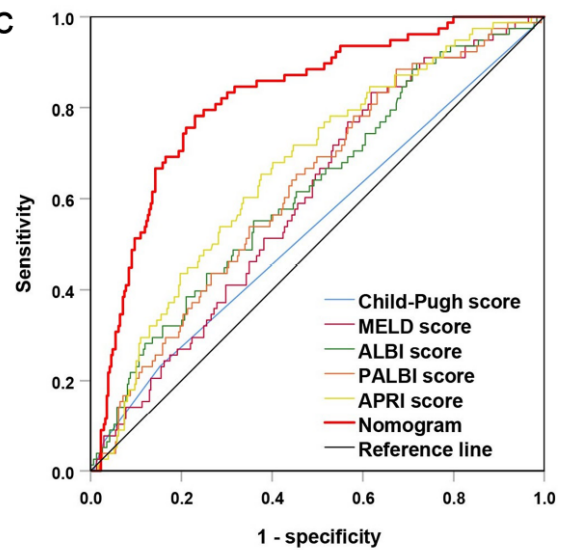

Figure 4

A: Total points distribution of false positive events (blue polyline). The $X$-axis represents the total points used to predict the risk of grade $B / C$ PHLF, the $Y$-axis represents the frequency of false positive events. The red dotted line represents the fitted line and presents a normal distribution. a. training cohort, the false positive events were concentrated around the maximum value 175 point, and close to the preset cutoff (169 points). b. Internal validation cohort, the false positive events were concentrated around the maximum value 170 points. c. External validation cohort, the false positive events were concentrated around the maximum value 176 points. B: Comparison of predicative performance for predicting grade B/C PHLF between the nomogram and conventional scores: a. training cohort, b. internal validation cohort, c. external validation cohort.

\section{Supplementary Files}

This is a list of supplementary files associated with this preprint. Click to download.

- supplementalTable2.docx

- SupplementalTable1.docx

- supplementalTable2.docx

- SupplementalTable1.docx 